\title{
Research on the Teaching Mode of Idioms in International Chinese Language
}

\author{
JungChih Tsai ${ }^{1, a^{*}}$, ChingChih Tsai ${ }^{2, b}$ \\ ${ }^{1}$ School of Humanities, Minjiang University, Fuzhou City, Fujian Province, China. \\ ${ }^{2}$ Institute of Applied English, National Taiwan Ocean University, Keelung, Taiwan \\ a*jungchih99@qq.com \\ bching2005@gmail.com
}

\begin{abstract}
For the idioms teaching of Chinese as a second language, scholars have proposed various teaching strategies, most of which focus on how to explain the meaning of idioms, using interesting and contextual methods, or online videos for teaching. There is very little emphasis on the sentence structure of idioms and the design of teaching experiments. This article proposes to analyze the structure of idioms, design two types of four groups of teaching experiments in this respect, and propose an effective teaching mode of idioms.
\end{abstract}

Keywords: International Chinese Language, idioms, Teaching model

\section{国际汉语成语教学模式研究}

蔡蓉芝 $^{1, a *}$ 蔡菁芝 $^{2, b}$

1 闽江学院人文学院, 福州, 福建, 中国

2 海洋大学应用英语研究所, 基隆, 台湾

a*jungchih99@qq.com

${ }^{b}$ ching2005@gmail.com

\section{摘要}

针对国际汉语成语教学, 学者提出各种教学策略, 多数集中在如何解说成语的意义, 运用趣味化与情境化方 式, 或者网络视频等进行教学, 强调成语句法结构、设计教学实验等很少, 本文针对这方面提出分析成语结 构、设计两类四组教学实验, 提出有效的成语句法教学模式。

关键词: 国际汉语，成语，教学模式

\section{1. 引言}

成语研究在二十世纪中期已经开始, 迄今超过 半个世纪, 研究的重点有: 1. 成语本体研究 2. 学习 者偏误分析 3. 词典编纂 4. 多媒体教材开发 5. 教学 策略与练习设计。针对教学策略的研究, 学者们提 出: 趣味性、情境性、文化性教学策略的运用, 建 议的教学方式如: 可根据同义、反义等意义分类归 纳讲解, 讲解成语的句法结构, 配合语境且加强成语 的搭配训练, 运用教学视频。另有将对举成语当成 状态词教学, 并结合语素教学, 也有针对越南学生 的成语教学设计实验, 将汉越半音译成语透过对比
的方法加强教学。对于成语释义方面的研究是最多 的, 强调句法结构来教学、设计教学实验等就相对 的不足。本研究将以成语自身结构与句法结构结合 教学实验, 提出有效的国际汉语的成语教学模式。

\section{2. 成语结构分析}

成语是一种熟语, 有其固定的限制, 有可考 性、完整性、固定性、简练性等。如能了解成语的 组成来源, 有助于理解成语; 如能了解成语的组成 结构，则有助于学习如何在句子中使用成语。本文 以诗经中的成语为语料进行三方面的分析：组成来 
源、句法结构、意义结构。

\section{1. 组成来源}

以诗经成语为语料, 归纳出五类的成语来源:

直接从原文中抽出的四字:〈关睢〉：悠哉悠 哉、辗转反侧。〈大明〉：维此文王、小心翼翼。

（1）从原文中节缩而来：〈常棣〉：兄弟阅于墙一 “兄弟阅墙”。〈淇奥〉: 如切如磋, 如环如磨一 “切 磋琭磨”。 楚楚”

(2)变化原文而来:〈蜉蝣〉: 衣裳楚楚一“衣冠

（3）从原文中节缩变化:〈鹊巢〉: 维鹊有巢、维 鸠居之一“扸占鹊巢”。〈雄雉〉: 不忮不求, 何用 不臓, 《易·系辞下》: 人谋鬼谋, 百姓与能一 “人谋 不䟱”（从不同原文中节缩组合）

（4）从原文中增字而来: 〈角弓〉: 此今兄弟, 绰 有余一 “绰有余裕”。

表一是诗经中成语组成来源五个类别的比例, “从原文直接抽出” 形成的成语占 $50 \%$, 是最多 的，其次是从原文中节缩，占 $21.74 \%$

表 1 诗经中成语组成来源

\begin{tabular}{|l|l|r|r|}
\hline 类别 & 个数 & \multicolumn{2}{|c|}{ 比例 } \\
\hline $\begin{array}{l}\text { 从原文直接 } \\
\text { 抽出 }\end{array}$ & 46 & \multicolumn{2}{|c|}{$50 \%$} \\
\hline \multirow{2}{*}{$\begin{array}{l}\text { 从原文中节 } \\
\text { 缩 }\end{array}$} & 单句 4 & $4.34 \%$ & \multirow{2}{*}{$21.74 \%$} \\
\cline { 2 - 3 } & 双句 15 & $16.3 \%$ & \\
\cline { 2 - 3 } & 三句 1 & $1.08 \%$ & \\
\hline 变化原文 & 13 & $14.13 \%$ & \\
\hline $\begin{array}{l}\text { 从原文中节 } \\
\text { 缩变化 }\end{array}$ & 6 & \multicolumn{2}{|c|}{$6.52 \%$} \\
\hline $\begin{array}{l}\text { 从原文中增 } \\
\text { 字 }\end{array}$ & 直接增字 1 & $1.08 \%$ & \multirow{2}{*}{$7.61 \%$} \\
\cline { 2 - 3 } 与它书结合 6 & $6.5 \%$ & \\
\hline
\end{tabular}

\section{2. 句法功能分析}

成语的组成结构, 以诗经成语为例, 可以归纳 出下列八类:

（1）主谓结构: 如 “鸭占鹊巢”、“人才济济”

(2) 动宾结构: 如 “不愧屋漏”、“率由旧章”

(3) 偏正结构: 如 “弄璋之喜”、“踝踝独行”

（4）并列结构: 如 “临深履薄”、“竹苞松茂”

（5）重迭: 如 “战战兢競”、“兢兢业业”

（6) 述补结构: 如 “求之不得”、“忧心忡忡”

（7）兼语结构: 如 “执柯作伐”

(8) 特殊结构: 如 “爱莫能助”、“筑室道谋” 下表二是诗经成语组成结构的比例, 以主谓结
构的成语最多，占 $40 \%$; 第二是并列结构，占 $29 \%$, 并列结构再分析为各类结构, 其中的主谓结构占总 数的 $7.6 \%$, 因此, 主谓结构的成语加上这部分, 占 总数接近 $48 \%$ ；第三是偏正结构，占 $16 \%$ 。

表 2 诗经成语的句法结构

\begin{tabular}{|c|c|c|}
\hline 语法结构 & 数量 & 比例 \\
\hline 主谓结构 & 37 & $40.22 \%$ \\
\hline 动宾结构 & 5 & $5.43 \%$ \\
\hline 偏正结构 & 15 & $16.3 \%$ \\
\hline 并列结构 & 27 & $29.35 \%$ \\
\hline 重迭 & 2 & $2.17 \%$ \\
\hline 述补结构 & 3 & $3.26 \%$ \\
\hline 兼语结构 & 1 & $1.08 \%$ \\
\hline 特殊结构 & 2 & $2.17 \%$ \\
\hline
\end{tabular}

\section{3. 意义结构}

成语的意义结构可分为三类: 相对义、类近 义、同义。

\subsection{1. 相对义: 有十类}

（1）日月相对: “日居月诸”

（2）日夜相对: “夙兴夜寱”

(3) 前后相对: “跋前軍后”

（4）刚柔相对: “吐刚茹柔”

（5）上下相对: “出谷迁乔”

（6）文武相对: “允文允武”

（7）厚薄相对: “临深履薄”

（8）远近相对: “室迩人远”

（9）深浅相对：“深厉浅揭”

（10）时间长短相对：“一日三秋”

\section{3. 2. 类近义:}

如 “不稼不稿”、“竹苞松茂”

\subsection{3. 同义:}

如 “辗转反侧”

诗经中的成语相对义的组成最多，藉由事物的 对比来呈现某种寓意的语言方式，在诗经中就已被 广泛使用。将成语的来源厘清, 依照组成结构分 析, 在教学时, 更能协助学生理解记忆, 教师在选 择先教哪些成语时，可根据组成来源、句法结构, 篮选比例较高的类别来进行教学。 


\section{3. 教学实验设计}

在厘清成语来源与结构后, 设计教学实验, 第 一组为 “意义典故组”, 教学时, 强调讲解成语的 典故与意义; 第二组为 “结构功能组”，除基本释 义，并加入句法结构的教学。

\section{1. 意义典故组}

意义典故组两组, 各 6 人、10人，东方、西方 学习者都有, 学习中文四年以上。每学期教学时 间: 16 次计 16 小时, 各持续进行两个学期。

教学方式: 教师课前提供学习材料, 从成语典 筛选出常用的成语, 一次提供 20 个成语, 学生事先 准备, 课堂上负责讲解 2-3 个成语, 说明典故或意 义、例句。学生发问, 教师协助回答。这是比较传 统的教学模式, 但是加入学生的自主学习, 不是完 全依赖教师讲解。 考题

成就测验：20个考 10 题单选题, 考完即讨论

学习结果: 亚洲学生较无疑问, 有成语学习背 景, 西方学生经常对答案感到困惑, 10 题单选测

验, 日韩学生通常答对 8-10 题, 欧美学生通常答对 6-7 题, 有时更低。

\section{2 . 结构功能组}

结构功能组两组, 各 1 人、 12 人。个人组是西 方学习者, 学习中文 4 年半, 教学时间: 16 次共 8 小时进行一个学期; 12 人组, 亚洲学习者, 学习中 文 3-5 年, 教学时间: 16 次共 24 小时。

教学方式: 个人组每次学习 10 个成语, 教师解 释成语意义, 句法结构, 学生造句并提问。12人 组, 老师先进行句法教学 3 次, 才进入每次学习 12 个成语, 每个学生负责讲解一个, 并提供例句, 其 他学生可提问, 老师协助解答, 进行一个学期, 学 完 60 个成语测验一次。下面举一个学生提问, 教师 回答的例子：学生造句 “他通过千辛万苦...” , 教师 修正为 “他历经千辛万苦”, 说明: 中文用 “历 经”, 表示经验, “通过” 是用于测验、考试; 把 “历经” 删除, “千辛万苦” 成为 “谓语” ，如:

“他千辛万苦，…”。

成就测验: 个人组未进行考题测验, 藉由例句 提问, 让学生回答成语在句子中的句法功能。团体 组则进行单选考题前测与后测, 两次各测验 60 题。

学习结果: 个人组在学习两个月后就表示没有 困难了, 每次例句的句法功能判断都正确无误。团 体组的两次前测与后测成绩, 后测进步 28\%、38\%, 第二次明显进步更多。

\section{4. 成语教学模式建议}

从教学实验里发现, 学生理解成语没有问题, 但是如何正确使用, 却有难度。如果只学习了意 义、典故, 看了成语视频, 也比较了相似词, 相反 词等, 在使用时仍然出现问题, 其实就是不知道成语 可以放在句子中的什么位置, 担任某种句子成分。 所以, 本文据此提出成语的教学模式建议。

\section{1. 意义典故教学适合有成语背景者}

根据前述的教学实验, “意义典故组” 的教学方 式比较适合有成语概念背景的日、韩学习者, 因为 日、韩深受中华文化影响, 他们也懂许多中国的成 语, 所以学习成果很好。对于西方学习者, 即使从 典故加强释义, 测验结果也不理想, 学习者仍然对 于考题有许多疑问。

\section{2. 结构功能教学适合没有成语背景者}

结构功能组的个人组是西方学生, 欧洲人, 由 于是个别教学, 学习成效高出团体教学组。当然, 这与预习、教学时间都有关, 个人组的学习者, 每 次都会预习 10 个成语, 团体组的学习者, 每周只需 讲解一个, 其他成语, 他们基本上不会花很多时间 预习, 只会抄写成语定义, 不见得理解那些成语 了, 等着上课时其他同学讲解。团体组中有 4 个人 学习中文在 4 年以上, 跟个人组比较接近, 在第二 次的测验, 平均答对 $95 \%$ 的考题。

\section{3. 成语句法教学模式}

经过前述的教学实验, 本文提出理想的成语教 学模式建议, 在学习成语的基本意义或典故来源 后, 说明使用的情境, 再强调成语的句法功能教 学, 让学习者理解如何使用成语在各种句子中。

（1）篮选常用成语作为教学材料, 可从小学生 写作辞典与中学生成语典篮选, 搭配词频搜寻常用 度, 也可以再依据成语结构, 多选择主谓、并列、 偏正等比例较高的成语, 并且是直接由原文节录出 来的成语, 然后决定要教学的成语顺序。教学材料 包含成语、释义、例句、句法功能、相似词、相反 词。

（2）教师讲解成语组成结构, 如: 主谓、动 宾、偏正、并列等

（3）教师讲解成语在句子中充当的句子成分, 以明确的例句说明成语在句子中当谓语、宾语、状 语、定语、补语的情形。如: “一望无际” 的句法功 能, “水天辽阔, 二望无际” , 担任谓语。“一望无 际的海洋”, 担任定语。又如 “三番两次”, “他三 番两次地告状” , 担任状语。“他三番两次的保 证, 都不能使我安心”, 担任定语。“讲过三番两 次, 他都不放心上”, 担任补语。再如 “千言万 
语”, “我有千言万语想跟他说…”, 担任宾语。

（4）分配学生预习要讲解的成语, 学生讲解时, 必须说明意义典故, 并且提供 2-3 种该成语担任不 同句法成分的例句。

（5）同学可提问, 如果负责讲解的人无法清楚 回答, 则由教师协助说明, 并且教师协助分析该句 法成分, 教师再提供例句让学生判断。

（6）相似词，相反词不建议特别强调，避免造 成学习负担。这个部分作为提供学习者参考的资 料, 只有出现常用的相似词再简易说明。

（7）练习方式要多样, 可提供情境、词汇让学 生练习造句, 将一个成语的各种句法功能都尝试练 习, 还要能判断该成语的句法功能。

（8）进行成就测验，教师根据每个成语的常用 句法功能设计考题, 题型可以是选择、造句, 也可 以是句子改写, 变换成语的句法功能。根据测验结 果, 再加强学生错误比例较高的成语进行说明练 习。

\section{5. 结论}

学习成语, 除了提升阅读能力外, 就是要在合 适的情境下, 正确的在句子里使用。本文以诗经里 的成语为语料, 先分析成语的结构, 再从四组的教 学实验中发现, 完整的成语释义、典故说明、词语 对比等, 仍不足以让学习者真正学会运用成语, 学 习者只停留在理解意义的层次。所以本文建议的成 语教学模式为 “成语句法教学模式”, 透过成语充 当的句子成分分析, 来达到全面理解成语的使用, 了解一个成语可以在句子中哪些位置出现, 如何搭 配其他的句子成分。许多成语具备两三种句法功 能, 透过加强这个方面的练习, 学习者更容易学会 如何正确无误地运用成语。

\section{REFERENCES}

[1] Huang, C.H. (2020) Talk about the Idioms Teaching of Teaching Chinese as a Second Language. J. Science \& Technology Information., 3:102-103.

[2] Huang, N.N. (2020) A Study on the Teaching Strategies of antithetical Idioms for Advanced Students. Master Thesis of Jilin University.

[3] Jiang, W.Q., Chen, A.W. (1982) On the Internal Order of Fixed Words in Parallel Structure. J. Contemporary Rhetoric. 4.

[4] Ruan, H.Y.(2020) Study on the teaching methods of idioms for Vietnamese students- Take "Hanyu Jiaocheng " (Vietnamese Compiled Edition) as an example. Master's Thesis of Minzu University of China.

[5] Shih, L. (2008) Error Analysis and Teaching Strategies of Foreign Students' Use of Chinese Idioms. J. Journal of Southwest University for Nationalities (Humanities and Social Sciences Edition)., 6:280-283.

[6] Shen, L. N. (2007) A Summary of the Idioms Teaching in Teaching Chinese as a Foreign Language in the Past Ten Years. J. Journal of Language and Literature Studies., 7:158- [7] Tang, X.W., Zhao, L.J. (2020) Analysis of Misuse and Discussion on Teaching Strategies of Idioms in Teaching Chinese as a Foreign Language. J. "Journal of Qiqihar University" (Philosophy and Social Science Edition). 1:171 -173.

[8] Wang, T. (1995) Comprehensive Dictionary of Idioms. Shanghai Lexicographical Publishing House, Shanghai.

[9] Bai, X.J. (2020) Research on Teaching Chinese Idioms as a Foreign Language. J. Journal of Henan Institute of Education (Philosophy and Social Sciences Edition)., 3:119-124.

[10] Zhu, J.N. (1999) Chinese Lexicology. Wu-Nan Book Inc, Taipei.160. 